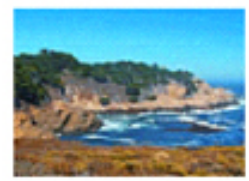

Western Medical Research Conference

(formerly Western Regional Meeting)

January 20-22 2022

Carmel, California

Western Section A merican Federation for Medical Rese arch

Western society for clinical Investigation

Western Association of Physicians

Western Society for Pediatric Research

Western Student and Resident Medical Rese arch Forum

\title{
2022 Western Medical Research Conference
}

Award Winners

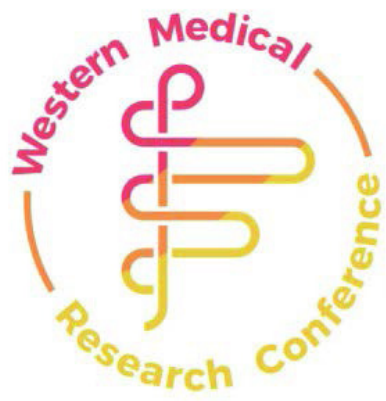

AFMR Henry Christian Award

Fatima Alduraibi, University of Alabama at Birmingham, VA Clinic Birmingham

Emily Batton, University of California San Diego

Erin Finn, University of Colorado, Children's Hospital Colorado

Kaidi He, Children's Hospital of Orange County

Slavita Laies, University of New Mexico

Schuyler Tong, UCSF Benioff Children's Hospital Oakland

Edwin E. Osgood Award

Kim Vo, Western University of Health Sciences

WAFMR Outstanding Investigator Award

Syed Bukhari, Temple University

WAFMR/WAP Student Subspecialty Award

Joni Aoki, University of Utah Health

Charlotte Archuleta, The University of Arizona College of Medicine Phoenix

Sang Hee Choi, Loma Linda University School of Medicine

Claire Conklin, University of California San Diego School of Medicine

Chase Czaykowsky, Loma Linda University School of Medicine

Nathan Davies, University of Washington School of Medicine, University of Idaho

Cameron Fateri, University of California Irvine

Julie Giannini, University of New Mexico School of Medicine

Jackie Hu, Western University of Health Sciences College of Osteopathic Medicine of the Pacific-Northwest

Jake Plagenz, University of Washington School of Medicine,

Shera Wanner, University of Washington School of Medicine

WAFMR/WAP/WSCI Outstanding Resident Award

Lankai Xu, University of California Davis Health System

WAP/WSCI Travel Award

Sami Dwabe, University of Southern California Medical Center, Keck Hospital of USC

Kendra Francis, Seattle Children's Hospital, University of Washington School of Medicine

Alana Freifeld, University of Colorado Denver

Donna Lien, Loma Linda University Adventist Health Sciences Center

Dante Mesa, University of Colorado

Kathleen Miao, NYU School of Medicine, Cornell University

Ozgur Oto, University of Colorado Health

Samantha Ratnayake, Kern Medical Center 
Matthew Rockstrom, University of Colorado

Arita Thatte, University of Washington

WSPR Abbott Nutrition David W. Smith Pediatric Trainee Research Award

Rebecca Levy, Stanford University School of Medicine

WSPR Abbott Nutrition Lowell Glasgow Student Research Award

Kathryne Foos, University of New Mexico Health Sciences Center

WSPR Abbott Nutrition Outstanding Resident Research Award

Jorge Rodriguez-Gil, Stanford University School of Medicine

WSPR Abbott Nutrition Subspecialty Award

Stephanie Chassen, University of Colorado

Danielle Cooke, University of Colorado Denver School of Medicine

Evan Giusto, University of California Davis

Alison Schildt, Stanford University

Christina Tise, Stanford University

Brandon Tomlin, University of Utah Health

Lindsey Trinh, Charles Drew University of Medicine and Science

WSPR Abbott Nutrition Young Investigator Research Award

Jason Nagata, University of California, San Francisco

WSPR Reckitt/Mead Johnson Nutrition Junior Faculty Award

Timothy Bahr, Intermountain Healthcare, University of Utah Health

Sandra Leibel, University of California San Diego

Megan Louderman, University of Colorado

Alicia White, University of Colorado

Kristen Wigby, University of California San Diego, Rady Children's Institute for Genomic Medicine

WSPR Reckitt/Mead Johnson Nutrition Travel Award

Thomas Christensen, University of Utah Health

Brian Lee, University of California Los Angeles

Sierra Levene, University of Washington School of Medicine

Juan Ramos, Stanford Medicine

Andrew Rebentisch, The University of Utah School of Medicine

Evan Scholten, University of Washington School of Medicine

Ana Starcevich, University of California Davis School of Medicine

\section{WSPR Prolacta Bioscience Research Travel Award}

Kati Bailli, University of New Mexico

Adrienne Cohen, University of Utah Health

Marie Cornet, University of California San Francisco

Laura Forero, University of California San Diego

Shawn Hanson, Loma Linda University

Emma Harding, University of Colorado

Brandon Lieu, Stanford University

Vanina Taliercio, University of Utah Health 